\title{
Remote Operation of the Fermilab/NICADD Photoinjector
}

\author{
N. Barov*, K. Desler, D. Edwards, R. Flora ${ }^{\ddagger \S}$
}

March 11, 2002

\footnotetext{
* Northern Illinois University NIU

${ }^{\dagger}$ Deutsches Elektronen-Synchrotron DESY

${ }^{\ddagger}$ Fermi National Accelerator Laboratory FNAL

$\S$ FNAL is operated under contract with the US Department of Energy
} 


\section{Introduction}

The recognition that a new major HEP facility must receive international support and that its execution would benefit from worldwide interaction from design through operation has given rise to the term Global Accelerator Network (GAN)[1].

A welcome consequence has been a more permissive attitude toward remote operation of existing facilities. For roughly thirty years, the computer has been the principal operator interface to the beam, and, through the development of the Internet, the operator can be literally anywhere.

In this note, we describe our approach to enabling a sufficient degree of operation of the photoinjector from afar in order to carry out investigations in beam physics. Our goal was to do so on a time scale of a few months and at minimal cost.

At this writing, remote shifts are routinely scheduled involving DESY and LBL, limited in frequency only by the requests of the collaborators and by the need for time to interpret the data.

\section{Description of the Photoinjector}

The photoinjector was designed to produce an electron beam with the bunch intensity and pulse structure of the TESLA parameters as they were some years ago[2]. Many components of the injector for the TESLA Test Facility (TTF) linac at DESY are identical and were provided from the same sources. A more detailed description is given by Carneiro[3].

The electron source is an RF photoemission gun which yields electrons with a kinetic energy of about $4 \mathrm{MeV}$. Immediately downstream, the electrons enter a superconducting booster cavity of the TESLA design from which they emerge with an energy in the 15-17 MeV range. The last major injector element is a magnetic bunch compressor.

Downstream of the bunch compressor, some 10 meters of beam line are available for diagnostics and experiments. Distributed throughout the beam line are a dozen quadrupoles and OTR view screens, and three slit systems for emittance measurement. At the end of the line, energy analysis may be performed by a magnetic spectrometer.

The CsTe cathode of the electron gun is illuminated by UV from a laser which is itself a research instrument[4]. It was not designed with remote adjustment in mind, and its performance is very sensitive to local conditions not easily monitored because of the complexity of the system.

High RF power for the gun and booster cavity is provided by modulators and klystrons that antedate any thought about remote control, but the low level RF control is easily accessible. Fortunately, the turn-on of the modulators and other equipment for which there are safety considerations needs only to be done once in a shift.

The control room for the photoinjector rather resembles an antique computer museum; a collection of PC and Mac castoffs, and a couple of Sun workstations, obtained at no cost and running a variety of operating systems. The one piece of elegant equipment, a LeCroy digital scope, monitors timing of the laser, timing and amplitude to the gun and booster cavity RF, and bunch charge. We think it is important to note that this hodgepodge did not present serious impediment to what we were trying to do. 


\section{Remote Operation of Experiments}

A new facility can be designed with remote operation in mind from the outset, and that model is fine for the future. Insofar as valuable beam physics $R \& D$ in the near term is concerned, we feel that can be accomplished with remarkable ease in a pre-existing facility.

The cutline between remote access and reservation of local control is important. Manipulation of the beam remotely to perform a measurement is essential, but turn on of a modulator is not. Laser tuning would be desirable, however that would require both remote expertise and a local hardware investment beyond the scope of the activity reported here.

Therefore the model is one in which the remote experimenters carry out their procedures, but every now and then they must ask someone in the photoinjector control room to do something not yet accessible to them. This has turned out to be a reasonable arrangement. For example, a 4 PM to midnight shift at DESY corresponds to 9 AM to 5 PM at Fermilab, during which period the photoinjector control room is normally populated and so requests from abroad are easily handled.

In the subsections below, we comment on the three systems in which changes were necessary. The Fermilab electronic logbook already existed; remote entry needed only a permission. Voice and visual interaction among experimenters is carried out using H323 protocol [9] compatible videoconference tools. Cryogenics for the superconducting booster cavity normally needs attention only at the beginning and end of a shift, and so no special provision was needed.

\subsection{Settings and Readings}

Many adjustments of magnet currents, motor positions, low level RF control and the like are performed from a computer screen, and are very low bandwidth activities. Such programs as VNC[5] and Timbuktu[6] enable the presentation of a local screen anywhere else in the world, provided the appropriate permission is granted. VNC is free, and is in use for most of these purposes at the three laboratories in the present collaboration. An exception is the Sun workstation used for low level RF control; it is accessed via ssh.

\subsection{Video Switching}

Profiles of the laser and electron beams are obtained from 19 CCD cameras which view a variety of screens. For profile recording, a mechanical push-button actuated video switch was the original device to direct the video sources to a frame grabber. Two 16 input to 1 output switches controllable via RS232 were purchased from RF Systems, Inc.[7] to permit remote operation through an existing Internet Rack Monitor[8]. The oscilloscope is viewable remotely by connecting its VGA output to an input of a video switch.

\subsection{Video Serving}

Video data is generated at the rate of $300 \mathrm{~KB} / \mathrm{s}(640 \times 4808$-bit grey scale pixels at $1 \mathrm{~Hz})$. To conserve network resources and minimize the time lag for viewing an image, compression is used during transfer. The lossy JPEG format has proven to be an excellent way to present video information for one-time viewing by an operator. At the other end of the scale, the zlib algorithm[10] offers lossless compression for data analysis and archiving. 
The images are first captured with a Scion LG-3 frame grabber card[11], and converted to JPEG using the cjpeg program[12]. A compression ratio of 10:1 is typical for this type of data, hence a 30 $\mathrm{KB} / \mathrm{s}$ data rate. The images are then distributed with the Apache web server using the server push method. The auxiliary program that drives the server push was modified from its original form[13] to receive its input from the frame grabber, as well as to optionally rescale the brightness level as specified by the user.

The goal for remote data analysis was to make the data easily available to high-level programming environments such as Labview and Matlab on the remote end. A custom protocol was developed for this purpose[14]. The remote end first specifies a number of options, such as the region of interest, the frame rate, and whether or not to use compression. The server then periodically sends the video data, and an optional set of additional variables such as the timestamp and shot index value. The data must be decompressed with zlib, which can be compiled on a wide range of computing platforms.

\section{Concluding Remarks}

We feel that this approach to remote experimental involvement is working very well. Total expenditure for purchases at Fermilab for this purpose has been less than $\$ 3000$ to date. We do not have plans for significant further development in the near term; it is necessary to exploit the present capability. There are suggestions for a major photoinjector upgrade, and, should that come to pass, comprehensive remote operation will be a consideration from the outset.

\section{Acknowledgements}

It is a pleasure to thank Bob Florian, Bob Goodwin, Gerhard Grygiel, Mike Kucera, and James Santucci for their essential contributions throughout this activity.

\section{References}

[1] TESLA Technical Design Report, TESLA 2001-23, March 2001, Editors F. Richard et al

[2] TESLA TEST FACILITY - TESLA Report 95-01, March 1995, Editor D. A. Edwards.

[3] J.-P. Carneiro, Etude Expérimentale du Photo-Injecteur de Fermilab, Universite Paris XI, 2001, http://fnalpubs.fnal.gov/archive/2001/thesis/t-carneiro.ps

[4] A. R. Fry, M. J. Fitch, A. C. Melissinos, B. D. Taylor, Laser system for a high duty cycle photoinjector, Nuclear Inst. \& Methods A 430, p. 180-188 (July 1999).

[5] VNC is freeware available at http://www.uk.research.att.com/vnc/

[6] Timbuktu is a product of Netopia Inc, http://www.netopia.com.

[7] R.F. Systems, Inc. The website is at http://www.rfsystems.com.

[8] Internet Rack Monitor, M. Shea, R. Goodwin, and M. Kucera, http://irm.fnal.gov/overview/irmwrt.html 
[9] http://www.openh323.org

[10] This library is available from http://www.zlib.org.

[11] The company can be found at http://www.scioncorp.com. The device driver used to communicate with the hardware in the Linux environment can be found at: http://www.sourceforge.com/projects/scionlg3.

[12] cjpeg is part of the GNU collection (http://www.gnu.org).

[13] http://www.esosoft.com/contrib/cgi/push/push.c

[14] To be published. 